\title{
Action observation in the modification of postural sway and gait: theory and use in rehabilitation
}

\author{
Mitesh Patel \\ Division of Brain Sciences, Imperial College London, Charing Cross Hospital, \\ London. W6 8RF, UK.
}

Correspondence: Mitesh.Patel1@imperial.ac.uk

\begin{abstract}
The discovery of cortical neurons responsive to both the observation of another individual's movement and one's own physical movement has spurred scientists into utilising this interplay for rehabilitation. The idea that humans can quickly transfer motor programmes or refine existing motor strategies through observation has only recently gained interest in the context of gait rehabilitation but may offer significant promise as an adjunctive therapy to routine balance training. This review is the first dedicated to action observation in postural control or gait in healthy individuals and patients. The traditional use of action observation in rehabilitation is that the observer has to carefully watch prerecorded or physically performed actions and thereafter imitate them. Using this approach, previous studies have shown improved gait after action observation in stroke, Parkinson's disease and knee or hip replacement patients. In healthy subjects, action observation reduced postural sway from externally induced balance perturbations. Despite this initial evidence, future studies should establish whether patients are instructed to observe the same movement to be trained (i.e., replicate the observed action(s)) or observe a motor error in order to produce postural countermeasures. The best mode of motor transfer from action observation is yet to be fully explored, and may involve observing live motor acts rather than viewing video clips. Given the ease with which action observation training can be applied in the home, it offers a promising, safe and economical approach as an adjunctive therapy to routine balance training.
\end{abstract}

Keywords: Action observation; postural sway; gait; rehabilitation; mirror neurons

\section{Introduction}

Falls are the leading cause of disability, hospitalisation and morbidity in patients with movement disorders, but effective balance training can mitigate fall risk. The general approach for effective training involves learning or re-learning 
dynamic multisensory tasks which provoke the development of motor strategies and central adaptation. The implementation of different rehabilitative techniques may therefore have a positive effect in preventing falls and reducing postural and gait instability in challenging environments.

Conventional balance or gait training involves assisting movements, preserving muscular tone and modifying current gait strategies with the main focus of reducing undesirable movements. Such approaches allow patients to maintain their motor potential and utilise their remaining motor abilities. However, rehabilitation is not always fully successful and consequently physiotherapists are looking towards adjunctive therapies.

Neurorehabilitation, in which the brain is directly stimulated in order to elicit structural and functional brain changes, can improve the outcome of routine balance training [1], but surprisingly little is known about the rehabilitative effects of action observation in relation to balance or gait training. This review will detail the underlying basis of action observation training, present findings from clinical and non-clinical action observation studies of postural sway and gait and provide suggestions for future study.

\section{The scientific basis of action observation}

Humans have an astonishing ability to predict another person's movements and make adjustments to motor plans. Why are we so good at reading another individual's movements, and can this be applied to balance training?

Classical studies indicate that human neonates, only a few days old, can imitate the motor actions of the parent such as sticking out a tongue [2] and infants below two years of age can predict the movement goals of another individual [3], suggesting that the brain is fortified with a mechanism linking action observation, understanding and imitation. The discovery of a population of neurons responsive to both the observation of motor actions and their execution in the macaque monkey [4] provided the mechanism by which the motor actions of others could be understood and mimicked.

However, it was not until later that a human equivalent, referred to as the 'mirror neuron system', was established using neurophysiological, neuropsychological and neuroimaging techniques [5]. The existence of mirror neurons was further enhanced by intracranial diagnostic recordings during craniotomy which showed that there are specific cortical neuronal populations in humans that respond to both the observation of another individual's actions (action observation) and one's own physical movements [6]. The belief is that action observation causes a mental, virtual re-enactment of the observed actions. 
Such an internal simulation makes it possible to reactivate action representations previously stored in motor memory, transfer new motor repertoires between individuals and train regions of the brain responsible for movement.

\section{Action observation and motor learning}

Motor learning is critical to the outcome of balance training and involves a number of components such as sensory processing, applying decision making strategies and initiating control processes based on prediction and biomechanical constraints. Once an action is learnt, we come to perform it effortlessly such as negotiating challenging terrains during locomotion by automatically adjusting our gait. Thus, by means of motor learning, we continuously extend our motor repertoire and generate new motor skills. For example, an everyday experience faced by commuters is negotiating a moving escalator which we do so by releasing a learnt predictive postural adjustment to compensate for the mechanical balance perturbation [7].

First evidence for the involvement of the mirror neuron system in motor learning was their discovery in the ventral premotor area (F5) of the macaque monkey [8]. Later studies in humans have revealed a complex network of mirror neurons in planning, strategic and motor areas $[9,10]$. By means of non-invasive techniques, it has been possible to show that action observation involves activation of specific regions of the frontal and parietal lobes; the posterior inferior frontal lobe (premotor), the ventral precentral gyrus, supramarginal cortex and rostral inferior parietal lobe $[6,11]$, but the distribution of mirror neurons is probably scattered. During observation of complex novel motor actions, neuronal populations have been found active at the exact moment of movement initiation until its completed execution, with specific contributions from the prefrontal cortex in reorganising existing motor repertoires [12] and motor cortical changes specific for the muscles involved in the observed action [13], coupled in terms of temporal coding and force requirements [14]. Such a distribution and specificity is concordant with the view that action observation is an effective way of developing motor strategies and building permanent motor memories [15]. Accordingly, experiments show that observing repeated thumb movements leads to a kinematically specific memory trace of the observed movements in the motor cortex [16]. In relation to whole body motor learning, Gatti and colleagues [17] showed that the observation of novel arm and foot movements is sufficient to produce new motor kinematics. It is therefore likely that action observation leads to organisational changes in the brain which may be directly associated with motor learning. 
Buccino and colleagues [1] propose four possible mechanisms where the mirror neuron system could be involved in developing new motor strategies or reestablishing previously learned motor skills: "1. Acoustic and tactile feedback enhances the acquisition of the observed motor action (i.e., conditioning). 2. The mirror neuron system has a direct influence on activation of corticospinal pathways. 3. The mirror neuron system activates previously learned movements from the stored repertoire. 4. Imagining movements that are observed may facilitate learning."

The next section reviews action observation studies with the aim of modifying postural sway or gait in healthy subjects.

\section{Literature Review}

In March 2017, PubMed was searched for English-language studies with the terms "action observation gait/postural control/locomotion/balance", "mirror neurons gait/postural control/locomotion/balance". Inclusion criteria were any physiological studies involving the observation of gait or posture either firsthand or from video recordings, and subsequent observer replication of locomotor or balancing tasks. No studies meeting criteria were excluded. After retrieving all material along this research theme, studies were divided into material on healthy subjects and those on patients, as below.

\subsection{Action observation in the control of postural sway and gait}

1. Can we mimic observed gait and postural responses of another individual in routine tasks?

The potential of the mirror neuron system in gait training was demonstrated in an early study, which found improved balance responses and gait in elderly individuals moving through an activity course after having first imagined their movement (motor imagery) [18], a method associated with the activation of cortical circuits involved in motor planning and programming (some of which are shared by action observation). The neurophysiological basis of these findings were supported by Behrendt and colleagues [19] who demonstrated that healthy subjects observing another person walking, recreate a mental representation of the observed gait which they reproduce concurrently in lower limb EMG recordings. Observing the sway of others can also affect current and future postural tasks, where subjects tend to mimic the observed individual's sway. For example, in a study by Taube and colleagues [20], participants observed videos of an actor performing balance exercises over four weeks. When observers were then asked to perform a balancing task on a free-moving platform, they employed the same techniques that they had observed and significantly reduced their postural sway in the task. 
2. Can we acquire anticipatory balance responses after observing gait and postural actions in challenging tasks?

Motor learning is the process in which motor strategies are formed or modified through practise, and in the context of balance control, is responsible for the development of anticipatory responses [1]. Thus, whether action observation can produce motor learning through the covert simulation of observed movements is crucial to the development of rehabilitation programmes. To that end, Patel and colleagues [7] sought to investigate differences between adaptive locomotor learning induced by first-hand experience versus action observation. Specifically, the authors studied whether observation of an actor's postural sway on a moving sled was sufficient to generate automatic compensatory postural responses. They found that after observing another individual swaying upon a sled that accelerated across a linear track, the observer produced a compensatory postural response in proportion to the size of the observed motion. The overall motor learning effect was similar to physically performing the task, but about $50 \%$ smaller. Crucially, observers generated no compensatory response after observing the sled move in isolation, suggesting that observing an actor was critical to motor learning. Similarly, Bhatt and Pai [21] showed that after observing another person slip because of a sudden unpredictable platform translation, subjects going on to perform the same experimental paradigm had lower slip displacement and velocity and greater post-slip stability compared to naïve subjects.

To summarise, updating cognitive centres with new gait patterns or postural consequences of an impending task through observation yielded tangible postural and gait benefits. It is therefore possible that observers generate new predictions about the task by covertly simulating the motor commands of the observed action. Nevertheless, observational learning is not as effective as physical motor learning when the two are compared. However, it should be pointed out that action observation may have advantages over physical task performance when individuals are asked to reproduce multiple motor tasks [22], but this is yet to be confirmed in gait learning studies.

\subsection{Action observation in gait rehabilitation}

The capacity of the mirror neuron system to take part in motor learning through action observation has only recently been applied to balance and gait rehabilitation [1]. In these studies the patient has had to carefully watch prerecorded actions and thereafter imitate them. To date, this approach has been applied in conjunction with routine rehabilitation in Parkinson's disease, postsurgical joint replacement and stroke patients. This section summarises published studies in this area. 


\section{Parkinson's disease}

In a randomised controlled study, Buccino and colleagues [23] investigated the effectiveness of action observation as a complementary therapy to pharmacological treatment and normal balance training in Parkinson's disease. In the study, patients in the experimental group observed videos depicting everyday actions, including balancing actions and walking, whereas those in the control group observed movies without specific motor content. After treatment, the experimental action observation group scored better on the unified Parkinson's disease rating scale and the functional independence measure than controls, but there was no objective difference in gait performance between groups. Similarly, Jaywant and colleagues [24] investigated the efficacy of homebased gait observation training to enhance walking in Parkinson's disease in a randomised controlled trial. Participants were divided into two groups, an experimental group who observed a video of archetypal gait, daily over 8consecutive days and performed routine balance training, and a control group who only performed balance training. After the training phase, the authors reported no difference in gait performance between the two groups but the experimental action observation group had a significant increase in selfperceived mobility (PDQ-39 mobility subscale). The authors suggested that the repetition of the same gait in the experimental video, without challenge or novelty, might explain their findings. In another study, Santamato and colleagues [25] performed a prospective open-label study of two-months action observation treatment in Parkinson's disease. The authors asked patients to observe a video of motor tasks linked to balance daily and then asked to replicate performance. The authors found no change of gait performance over time, but there was no control group. It is interesting to note that the basal ganglia are heavily connected with regions of the mirror neuron system such as those involved in motor planning and motor learning. Hence, sufficient dopamine may be a pre-requisite for action observation and help to explain these findings. Accordingly, a recent study by Pelosin and colleagues [26] of finger movements showed that action observation can improve movement velocity in Parkinson's disease but the effects are only retained 40 minutes after intervention treatment in patients ON L-dopa.

Despite the studies above which indicate little physical improvement in Parkinson's disease, Pelosin and colleagues have shown that action observation reduces freezing of gait (freezing of gait questionnaire and number of episodes) in Parkinson's disease [27]. In the study, the authors compared an experimental group who observed video clips showing specific movements and strategies to circumvent freezing of gait episodes and controls who observed a landscape scene in addition to routine physical therapy three times a week, for four weeks. Similarly, Agosta and colleagues [28] examined the efficacy of action observation training on disease symptoms, freezing of gait and motor abilities in Parkinson's 
disease. Patients were randomised into two groups, an experimental group who observed the gait of an actor over four weeks in addition to physical training and a control group who observed a landscape video in addition to physical training. The experimental action observation group showed reduced motor disability and improved balance scores at four weeks compared to controls. At eight weeks, improved motor ability, increased walking speed, balance and quality of life with a trend towards reduced freezing of gait compared to baseline were found in the experimental action observation group.

\section{Post-surgical joint replacement}

In a randomised controlled trial, action observation was used in post surgical orthopaedic patients for hip fracture or elective hip or knee replacement to improve gait [29]. All patients underwent conventional physiotherapy, but patients in the experimental group observed video clips showing actions related to the lower limbs whereas the control group was shown video clips with no motor content six days per week, over three weeks. All patients were asked to physically perform gait movements mimicking those observed by the experimental group. After treatment, the experimental group scored significantly better than control patients on functional independence and the Tinetti scale. Similarly, Villafane and colleagues [30] conducted a prospective clinical trial in patients recovering from total hip arthoplasty and compared gait performance between an experimental group who observed normal gait in addition to conventional physical therapy and a control group who were given written instructions of gait in addition to conventional physical therapy over 10 sessions. They discovered that both treatments improved range of motion and Tinetti scale scores, but subjective improvements were better in the experimental action-observation group. It should also be noted that Park and colleagues [31] found that the addition of daily action observation training to gait training over three weeks produced improved pain, stiffness, and gait function scores in the Western Ontario and McMaster Universities Osteoarthritis Index (WOMAC) after total knee replacement.

\section{Stroke}

Studies of action observation in stroke patients have produced highly positive results: Bang and colleagues [32] performed a double-blind randomised controlled trial in chronic stroke patients comparing gait performance of an experimental group who observed a video of treadmill walking actions at different speeds before routine training and a control group who observed a nature video before routine training five times a week, for four weeks. Timed upand-go, 10-metre walk, 6-minute walk and maximal knee angle in the swing phase were better in the experimental action observation group compared to controls. Similarly, Kim and Lee [33] studied gait performance in chronic stroke patients ( $>6$ months after hospitalisation) after observation of gait for five 30- 
minute weekly sessions over a four week period. The authors found improved timed up-and-go, faster gait velocity, slower cadence and greater single limb support of the affected side. In a randomised controlled trial of action observation training in chronic stroke patients with hemiparesis by Park and colleagues [34], patients were randomly assigned to an experimental group who observed video clips demonstrating four-staged ambulation training for 30minutes, three times a week for four weeks alongside gait training, and a control group who observed landscape pictures alongside gait training. After the four week intervention, gait measures were better in the experimental action observation group compared to controls. The experimental group had better scores in the 10-metre walk test, community walk test, improved activitiesspecific balance confidence and stride length, longer single-led support time and faster gait velocity. In another randomised controlled trial of gait performance in chronic stroke patients with hemiparesis [35], patients were divided into an experimental group who observed an actor's gait in four different situations and gait training versus a control group who observed landscape images and performed gait training. Both groups were trained for 30 minutes, three times per week, for a four week period. The authors found that the experimental action observation group had improved 10-metre walk test, figure of eight test and gait symmetry in the stance phase, whereas controls did not. Park and Hwangbo [36] studied static balance and gait in hemiplegic chronic stroke patients in an experimental group: patients allocated to observation of flatland gait, slope gait and stair gait and gait training and a control group: patients allocated to observation of a nature video and gait training. Both groups observed video clips for 20-minutes per day, five times per week, for eight weeks, alongside routine gait training. The authors found improved gait velocity, larger limits of stability and better gait ability in the action observation group compared to controls.

The results from patient-based studies (Table 1) suggest that action observation, as an adjunctive therapy, improves measures of gait and balance function following a brief period of rehabilitation. In Parkinson's disease, action observation appears to facilitate mobility and a reduction of freezing of gait, whereas after joint-replacement or stroke, action observation significantly improves gait and balance across a range of measures.

\section{Limitations}

Although limited in number, the few studies that have asked individuals to observe a movement error and then perform a postural or gait task, have shown positive effects. Another limitation is that no study has investigated the effect of action observation on performance of gait or postural actions with new parameters after a retention period to assess long-term motor learning. On a related note, other shortcomings in the literature include the lack of long-term retention studies to address whether action observation is suitable for 
rehabilitation or in different patient populations. Further research in the form of randomised controlled trials is necessary.

\section{Review summary and future directions}

Given the high personal and economic cost of falls and the high incidence of balance problems in patients referred for rehabilitation, methods that can improve the outcome of rehabilitation are sought after. A critical aspect of balance training is learning or re-learning anticipatory postural adjustments or adjusting gait patterns - both of which are heavily dependent on motor learning. However, motor learning in the context of walking is poorly understood. Gait must be flexible enough to accommodate different environments or constraints, yet automatic enough to avoid having to consciously focus on each step. This complex interplay is partly driven by cortical processes influencing both longlatency postural responses via corticospinal loops and short-latency postural responses via indirect communication with the brainstem. As the mirror neuron system is partly situated within motor cortical regions, action observation is likely to activate these same motor cortical networks and participate in modifying environmentally appropriate responses and engaging in their preselection. The general conclusion from findings in the literature was that action observation is involved in the motor learning process and could be an effective adjunctive treatment to conventional therapy in the rehabilitation of neurological and non-neurological conditions. It must be underlined however that studies carried out so far have involved small numbers of patients and larger clinical trials are necessary.

\section{Future directions}

The goal of action observation training in clinical gait disorders is to target the damaged neural structures or pathways that perform the impaired or lost actions. This mechanism may involve understanding the observed actions and their consequences. As mirror neurons exhibit a substantial degree of abstraction, the observer's own motor response could be influenced by specific features of the observed action, such as the direction of movement and its outcome. It is also likely that observing motor actions within one's own repertoire enhances the effect of motor training. In an elegant study, expert dancers empathised more strongly when they observed another dancer performing a familiar dance type, than a dancer performing an unfamiliar dance [37]. Furthermore, as responses of a subset of F5 motor mirror neurons are modulated by the distance between the observer and the observed actor [38], the effect of action observation might depend on the ability of the observer to engage with the action unfolding in front of them. Indeed, there is stronger reactivity of the human primary motor cortex during observation of live rather than videoed motor acts [39]. The strength of this effect can be seen in basic experiments which demonstrate a change in reflex gain during gait by merely 
observing of another person physically walking [19], but is yet to be studied in gait rehabilitation.

Also, as the mirror neuron system is involved in more refined cognitive aspects of action understanding, the ability to understand the intentions of others and their predicament may serve as a mechanism to transfer anticipatory postural responses or reactive reflexes. Studies in healthy subjects $[7,21]$ showed that observing the gait of another person in a challenging environment was sufficient to generate appropriate anticipatory postural countermeasures, implying the existence of an automatic mechanism for adapting locomotor behaviour to external challenges. It is possible that understanding the implications of false movements or the actor's predicament helped to drive motor learning in these studies. Caggiano and colleagues [40] found that responses of most mirror neurons were stronger if the observed motor act was associated with reward. The authors showed that the discharge from mirror neurons was influenced by the importance of the motor act to the observer - which might extend to avoiding a fall. This effect has been labelled the "adaptation model of the mirror neuron system" as it confers an evolutionary advantage for survival e.g., recognition of negative emotions including fear and disgust, development of protective reflexes and formation of complex social behaviours [11]. It is possible that motor error observation quickly transfers both motor and cognitive cues such as fear, reward or empathy to reinforce observational learning. Although trials involving action observation training are increasing, an important aspect is thus to study whether patients should observe the same movement to be trained (i.e., replication) or movement error.

\section{Conclusion}

Although the numbers of studies are limited, there is evidence to suggest that action observation training is beneficial to the restoration of postural and gait control. Given the ease with which action observation training can be applied in the home, it offers a promising, safe and economical approach as an adjunctive therapy to routine balance training.

\section{Conflicts of Interest}

The author has no conflicts of interest.

\section{Acknowledgments}

This work was supported by the National Institute for Health Research (NIHR) Imperial Biomedical Research Centre. The views expressed are those of the authors and not necessarily those of the NIHR. 


\section{References}

[1]. Buccino G, Solodkin A, Small SL. Functions of the mirror neuron system: implications for neurorehabilitation. Cogn Behav Neurol. 2006;19(1):5563.

[2]. Meltzoff AN, Moore MK. Imitation of facial and manual gestures by human neonates. Science. 1977;198(4312):75-78.

[3]. Meltzoff AN. Understanding the Intentions of Others: Re-Enactment of Intended Acts by 18-Month-Old Children. Dev Psychol. 1995;31(5):838850.

[4]. di Pellegrino G, Fadiga L, Fogassi L, Gallese V, Rizzolatti G. Understanding motor events: a neurophysiological study. Exp Brain Res. 1992;91(1):176180.

[5]. Iacoboni M, Woods RP, Brass M, Bekkering H, Mazziotta JC, Rizzolatti G. Cortical mechanisms of human imitation. Science. 1999;286(5449):25262528.

[6]. Mukamel R, Ekstrom AD, Kaplan J, Iacoboni M, Fried I. Single-neuron responses in humans during execution and observation of actions. Curr Biol. 2010;20(8):750-756.

[7]. Patel M, Roberts RE, Riyaz MU, Ahmed M, Buckwell D, Bunday K et al. Locomotor adaptation is modulated by observing the actions of others. J Neurophysiol. 2015;114(3):1538-1544.

[8]. Ferrari PF, Rozzi S, Fogassi L. Mirror neurons responding to observation of actions made with tools in monkey ventral premotor cortex. J Cogn Neurosci. 2005;17(2):212-226.

[9]. Järveläinen J, Schürmann M, Hari R. Activation of the human primary motor cortex during observation of tool use. Neuroimage. 2004;23(1):187-192.

[10]. Avikainen S, Forss N, Hari R. Modulated activation of the human SI and SII cortices during observation of hand actions. Neuroimage. 2002;15(3):640-646.

[11]. Rizzolatti G, Sinigaglia C. The mirror mechanism: a basic principle of brain function. Nat Rev Neurosci. 2016;17(12):757-765.

[12]. Buccino G, Vogt S, Ritzl A, Fink GR, Zilles K, Freund HJ et al. Neural circuits underlying imitation learning of hand actions: an event-related fMRI study. Neuron. 2004;42(2):323-334.

[13]. Urgesi C, Candidi M, Fabbro F, Romani M, Aglioti SM. Motor facilitation during action observation: topographic mapping of the target muscle and influence of the onlooker's posture. Eur J Neurosci. 2006;23(9):25222530.

[14]. Alaerts K, de Beukelaar TT, Swinnen SP, Wenderoth N. Observing how others lift light or heavy objects: time-dependent encoding of grip force in the primary motor cortex. Psychol Res. 2012;76(4):503-513.

[15]. Stefan K, Classen J, Celnik P, Cohen LG. Concurrent action observation modulates practice-induced motor memory formation. Eur J Neurosci. 2008;27(3):730-738.

[16]. Stefan K, Cohen LG, Duque J, Mazzocchio R, Celnik P, Sawaki L et al. Formation of a motor memory by action observation. J Neurosci. 2005;25(41):9339-9346. 
[17]. Gatti R, Tettamanti A, Gough PM, Riboldi E, Marinoni L, Buccino G. Action observation versus motor imagery in learning a complex motor task: a short review of literature and a kinematics study. Neurosci Lett. 2013;540:37-42.

[18]. Linden CA, Uhley JE, Smith D, Bush MA. The effects of motor imagery on walking balance in an elderly population. Occupational Therapy Journal of Research. 1989;9(3):155-169.

[19]. Behrendt F, Wagner H, de Lussanet MH. Phase-dependent reflex modulation in tibialis anterior during passive viewing of walking. Acta Psychol (Amst). 2013;142(3):343-348.

[20]. Taube W, Lorch M, Zeiter S, Keller M. Non-physical practice improves task performance in an unstable, perturbed environment: motor imagery and observational balance training. Front Hum Neurosci. 2014;8:972.

[21]. Bhatt T, Pai YC. Can observational training substitute motor training in preventing backward balance loss after an unexpected slip during walking? J Neurophysiol. 2008;99(2):843-852.

[22]. Larssen BC, Ong NT, Hodges NJ. Watch and learn: seeing is better than doing when acquiring consecutive motor tasks. PLoS One. 2012;7(6):e38938.

[23]. Buccino G, Gatti R, Giusti MC, Negrotti A, Rossi A, Calzetti S et al. Action observation treatment improves autonomy in daily activities in Parkinson's disease patients: results from a pilot study. Mov Disord. 2011;26(10):1963-1964.

[24]. Jaywant A, Ellis TD, Roy S, Lin CC, Neargarder S, Cronin-Golomb A. Randomized Controlled Trial of a Home-Based Action Observation Intervention to Improve Walking in Parkinson Disease. Arch Phys Med Rehabil. 2016;97(5):665-673.

[25]. Santamato A, Ranieri M, Cinone N, Stuppiello LA, Valeno G, De Sanctis JL et al. Postural and Balance Disorders in Patients with Parkinson's Disease: A Prospective Open-Label Feasibility Study with Two Months of Action Observation Treatment. Parkinsons Dis. 2015;2015:902738.

[26]. Pelosin E, Bove M, Ruggeri P, Avanzino L, Abbruzzese G. Reduction of bradykinesia of finger movements by a single session of action observation in Parkinson disease. Neurorehabil Neural Repair. 2013;27(6):552-560.

[27]. Pelosin E, Avanzino L, Bove M, Stramesi P, Nieuwboer A, Abbruzzese G. Action observation improves freezing of gait in patients with Parkinson's disease. Neurorehabil Neural Repair. 2010;24(8):746-752.

[28]. Agosta F, Gatti R, Sarasso E, Volonte M, Canu E, Meani A et al. Brain plasticity in Parkinson's disease with freezing of gait induced by action observation training. J Neurol. 2017;264(1):88-101.

[29]. Bellelli G, Buccino G, Bernardini B, Padovani A, Trabucchi M. Action observation treatment improves recovery of postsurgical orthopedic patients: evidence for a top-down effect? Arch Phys Med Rehabil. 2010;91(10):1489-1494.

[30]. Villafañe JH, Pirali C, Isgrò M, Vanti C, Buraschi R, Negrini S. Effects of Action Observation Therapy in Patients Recovering From Total Hip Arthroplasty Arthroplasty: A Prospective Clinical Trial. J Chiropr Med. 2016;15(4):229-234. 
[31]. Park SD, Song HS, Kim JY. The effect of action observation training on knee joint function and gait ability in total knee replacement patients. J Exerc Rehabil. 2014;10(3):168-171.

[32]. Bang DH, Shin WS, Kim SY, Choi JD. The effects of action observational training on walking ability in chronic stroke patients: a double-blind randomized controlled trial. Clin Rehabil. 2013;27(12):1118-1125.

[33]. Kim JH, Lee BH. Action observation training for functional activities after stroke: a pilot randomized controlled trial. NeuroRehabilitation. 2013;33(4):565-574.

[34]. Park HJ, Oh DW, Choi JD, Kim JM, Kim SY, Cha YJ et al. Action observation training of community ambulation for improving walking ability of patients with post-stroke hemiparesis: A randomized controlled pilot trial. Clin Rehabil. 2016.

[35]. Park HR, Kim JM, Lee MK, Oh DW. Clinical feasibility of action observation training for walking function of patients with post-stroke hemiparesis: a randomized controlled trial. Clin Rehabil. 2014;28(8):794-803.

[36]. Park EC, Hwangbo G. The effects of action observation gait training on the static balance and walking ability of stroke patients. J Phys Ther Sci. 2015;27(2):341-344.

[37]. Calvo-Merino B, Glaser DE, Grèzes J, Passingham RE, Haggard P. Action observation and acquired motor skills: an FMRI study with expert dancers. Cereb Cortex. 2005;15(8):1243-1249.

[38]. Caggiano V, Fogassi L, Rizzolatti G, Thier P, Casile A. Mirror neurons differentially encode the peripersonal and extrapersonal space of monkeys. Science. 2009;324(5925):403-406.

[39]. Järveläinen J, Schürmann M, Avikainen S, Hari R. Stronger reactivity of the human primary motor cortex during observation of live rather than video motor acts. Neuroreport. 2001;12(16):3493-3495.

[40]. Caggiano V, Fogassi L, Rizzolatti G, Casile A, Giese MA, Thier P. Mirror neurons encode the subjective value of an observed action. Proc Natl Acad Sci U S A. 2012;109(29):11848-11853. 


\begin{tabular}{|c|c|c|c|c|c|}
\hline Population & Study & Protocol & Intervention & $\begin{array}{l}\text { Sample } \\
\text { size }^{+}\end{array}$ & Main Outcome \\
\hline \multirow{5}{*}{$\begin{array}{l}\text { Parkinson's } \\
\text { disease }\end{array}$} & Buccino et al., 2011[23] & Single session & $\begin{array}{l}\text { Video clips of routine balancing and } \\
\text { gait actions and normal balance } \\
\text { training }\end{array}$ & 7 & Observation improved UPDRS and Functional Independence scores \\
\hline & Jaywant et al., 2016 [24] & $\begin{array}{l}8 \text { consecutive days of home- } \\
\text { based training }\end{array}$ & $\begin{array}{l}\text { Video of healthy and Parkinsonian } \\
\text { gait and routine training }\end{array}$ & 13 & $\begin{array}{l}\text { Gait observation significantly improved mobility on the PDQ-39 over controls but not } \\
\text { walking speed, stride length, stride frequency, leg swing time, or gait asymmetry }\end{array}$ \\
\hline & Santamato et al., 2015 [25] & $\begin{array}{l}\text { Three sessions a week for } 8 \\
\text { consecutive weeks }\end{array}$ & $\begin{array}{l}\text { Video of routine balancing and gait } \\
\text { tasks (e.g., obstacle negotiation) }\end{array}$ & 15 & No evidence of improved balance and posture from observation only \\
\hline & Pelosin et al., 2010 [27] & $\begin{array}{l}\text { Three sessions a week over } 4 \\
\text { consecutive weeks }\end{array}$ & $\begin{array}{l}\text { Video of movements and strategies } \\
\text { to circumvent freezing of gait } \\
\text { episodes and physical therapy }\end{array}$ & 10 & Observation significantly reduced freezing of gait episodes \\
\hline & Agosta et al., 2017 [28] & $\begin{array}{l}\text { Three sessions a week for } 4 \\
\text { consecutive weeks }\end{array}$ & $\begin{array}{l}\text { Video of movements and strategies } \\
\text { of gait transitions and physical } \\
\text { therapy }\end{array}$ & 12 & $\begin{array}{l}\text { Observation significantly improved motor disability scores, walking speed, balance and } \\
\text { quality of life at 8-weeks, with a trend toward a persisting reduced freezing of gait } \\
\text { severity }\end{array}$ \\
\hline \multirow[b]{2}{*}{$\begin{array}{l}\text { Joint } \\
\text { replacement }\end{array}$} & Bellelli G et al., 2010 [29] & $\begin{array}{l}\text { Six sessions a week, for } 3 \\
\text { consecutive weeks }\end{array}$ & $\begin{array}{l}\text { Video clips showing daily balancing } \\
\text { and gait actions (leg/trunk actions) } \\
\text { and physical therapy }\end{array}$ & 30 & $\begin{array}{l}\text { Observation improved functional independence for efficiency and motor scores and } \\
\text { Tinetti functional scores }\end{array}$ \\
\hline & Villafane et al., 2016 [30] & Ten sessions & $\begin{array}{l}\text { Video of functional balance and gait } \\
\text { exercises and physical therapy }\end{array}$ & 9 & $\begin{array}{l}\text { Observation improved pain, range of motion, quality of life, and gait features (Tinetti } \\
\text { Scale and Lequesne Index) }\end{array}$ \\
\hline \multirow{5}{*}{ Stroke } & Bang et al., 2013 [32] & $\begin{array}{l}\text { Five times a week over } 4 \\
\text { consecutive weeks }\end{array}$ & $\begin{array}{l}\text { Video of treadmill walking at } \\
\text { various speeds before treadmill } \\
\text { training for } 10 \text { minutes }\end{array}$ & 15 & $\begin{array}{l}\text { Observation significantly improved timed up and go test, } 10 \text {-m walk test, } 6 \text {-minute } \\
\text { walk test, and maximal flexed knee angle in the swing phase during walking }\end{array}$ \\
\hline & Kim and Lee 2013 [33] & $\begin{array}{l}\text { Five weekly sessions over } 4 \\
\text { consecutive weeks }\end{array}$ & Video of gait plus physical training & 10 & $\begin{array}{l}\text { Observation significantly improved Timed Up and Go, gait speed, cadence, and single } \\
\text { limb support of the affected side }\end{array}$ \\
\hline & Park et al., 2016 [34] & $\begin{array}{l}\text { Three weekly sessions over } 4 \\
\text { consecutive weeks }\end{array}$ & $\begin{array}{l}\text { Video clips of four-staged } \\
\text { ambulation training and physical } \\
\text { training }\end{array}$ & 12 & $\begin{array}{l}\text { Observation significantly improved } 10 \text {-m walk test, community walk test, and } \\
\text { activities-specific balance confidence and the spatiotemporal parameters (i.e. stride } \\
\text { length, single support, and velocity }\end{array}$ \\
\hline & Park et al., 2014 [35] & $\begin{array}{l}\text { Three weekly sessions over } 4 \\
\text { consecutive weeks }\end{array}$ & $\begin{array}{l}\text { Video clips of routine balancing and } \\
\text { gait tasks and physical training }\end{array}$ & 11 & $\begin{array}{l}\text { Observation significantly improved the } 10 \text {-m walk test, figure-of- } 8 \text { walk test, dynamic } \\
\text { gait index, and gait symmetry score in the stance phase }\end{array}$ \\
\hline & Park and Hwangbo 2015 [36] & $\begin{array}{l}\text { Five weekly sessions over } 8 \\
\text { consecutive weeks }\end{array}$ & $\begin{array}{l}\text { Video of normal gait, slope gait, and } \\
\text { stair gait and physical training }\end{array}$ & 20 & Observation significantly improved limits of stability and gait ability \\
\hline
\end{tabular}

Table 1: Summary of patient-based gait rehabilitation studies included in this review. ${ }^{+}$Notes the sample size in the investigational group. 